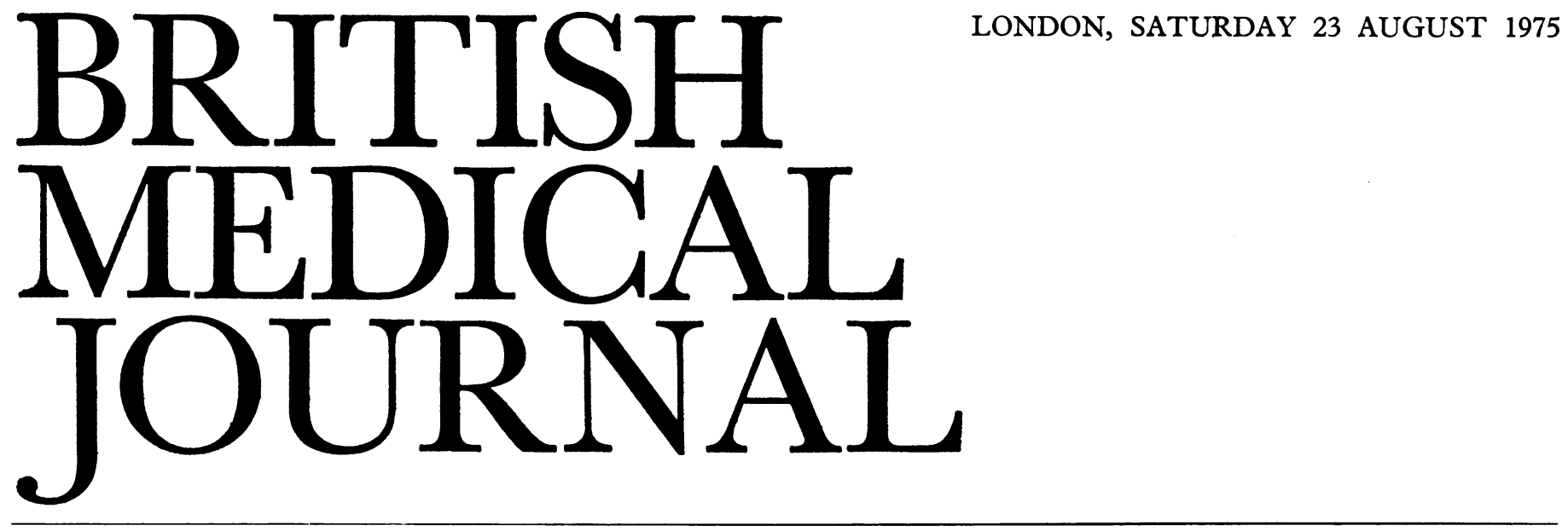

\title{
Low-dose Heparin and the Prevention of Venous Thromboembolic Disease
}

The past five years have seen an upsurge of interest in heparin given to prevent rather than to treat venous thromboembolic disease. As early as 1950 De Takats" wrote "it takes much less heparin ... to to prevent clotting than to treat it" and in 1970 Sharnoff and De Blasio ${ }^{2}$ reported in an uncontrolled trial that the perioperative death rate from thromboembolic disease was reduced approximately ten-fold by the prophylactic adminisstration of small doses of heparin.

A rational basis for the prophylactic use of small doses of heparin has emerged from recent studies of its mode of action, which have shown ${ }^{3}$ that it complexes with antithrombin III; the heparin-antithrombin II complex inhibits thrombin, but more powerfully inhibits factor $\mathrm{Xa}$ and other serine proteases in the coagulation sequence. ${ }^{45}$ Factor $\mathrm{Xa}$ has a key role in the sequence of events leading to the generation of thrombin, and indeed in animal experiments factor $\mathrm{Xa}$ was found to be a more potent thrombogenic agent than thrombin itself. ${ }^{6}$

These and other observations ${ }^{7}$ led to the concept of subcutaneous administration of heparin in low doses sufficient to inhibit factor Xa without prolongation of the whole blood clotting time or the production of a haemostatic defect of any clinical importance. Clinical validation of this concept became possible with the introduction of a satisfactory technique of leg scanning for deep vein thrombosis after the injection of radio-labelled fibrinogen, ${ }^{8}$ and it is now clear that low dosage heparin given subcutaneously at 5000 units twice or three times a day is effective in reducing the incidence of deep vein thrombosis as diagnosed by this technique in many surgical patients $^{9-13}$ and also probably in myocardial infarction ${ }^{13-15}$ though here the evidence is conflicting. ${ }^{16}$ As might be expected, the evidence in general suggests that 5000 units three times daily is both more effective in preventing thrombosis and associated with more bleeding problems than 5000 units twice daily. The major importance of a reduced incidence of deep vein thrombosis is that it should be associated with a reduced incidence of pulmonary embolism; a reduced incidence of the postphlebitic syndrome would also be expected, but might take many years to demonstrate. Browse et al. ${ }^{17}$ showed that in surgical patients positive leg scans correlated with changes in lung perfusion scans, and no reports have been published of pulmonary embolism in patients with negative leg scans. Lahnborg $s t$ al. ${ }^{18}$ carried out a double blind trial of low-dose heparin (5000 units two to five hours before surgery and then twice daily) with combined leg and lung scanning, and found a statistically significant reduction in positive lung scans; however, no control preoperative scans were carried out and there was a lack of correlation between positive leg and lung scans.

Recently, however, important direct evidence has come from an international multi-centre $\operatorname{trial}^{19}$ on the prevention of fatal postoperative pulmonary embolism by low-dose heparin. Surgeons in 28 centres participated in the study, which was co-ordinated at King's College Hospital, London. The originators of the study are to be congratulated on their pertinacity in seeing this large trial through to a successful conclusion. Patients over the age of 40 were selected for study; 2076 were randomly allocated to the control group and 2045 received calcium heparin 5000 units preoperatively and eight-hourly thereafter for seven days from single-dose ampoules. There was no laboratory control of the heparin therapy, and patients in the control group did not receive any specific prophylaxis. Patients were followed up until death or discharge from hospital. During the postoperative period there were 100 deaths in the control group and 80 in the heparin group; necropsy examinations were performed in $72 \%$ of the controls and $66 \%$ of the heparin patients. At necropsy, 16 patients in the control group and two in the heparin group were found to have died from acute massive pulmonary embolism $(\mathrm{P}<0.005)$. In addition, emboli were found at necropsy in six patients in the control group and three in the heparin group which were not considered to be the main cause of death. For all pulmonary emboli together the differences were again significant $(\mathbf{P}<0.005)$. As would have been predicted from the earlier studies ${ }^{9-13}$ the frequency of isotopically detected deep vein thrombosis in the 1292 patients in which the test was performed was reduced from $25 \%$ in the control group to $8 \%$ in the heparin treated group. The incidence of deep vein thrombosis as diagnosed clinically or by venography or at necropsy was also reduced by the heparin treatment.

The King's College study was also designed to get information on the incidence of bleeding. Detailed analysis of operative and postoperative bleeding was carried out in 1475 patients. No statistically significant differences in blood transfusion requirements or the fall of haemoglobin levels postoperatively were found between the heparin group and the controls. Five patients in the control group and four in the 
heparin group died from haemorrhage; but 158 in the heparin group as compared with only 117 controls developed wound haematomas.

In view of the importance of this study, detailed scrutiny of the trial design is proper. It is a pity that calcium heparin rather than the sodium salt was used; for, while it is likely that the sodium salt would have been equally effective and will probably be the form of heparin generally used in this country, direct proof of its efficacy in the prevention of fatal pulmonary embolism is lacking. While the necropsy rate (125 of 180 deaths) was high, the validity of the trial is critically dependent on the difference between 16 deaths from pulmonary embolism in the control group as compared with two deaths in the heparin group, and it is unfortunate that necropsies were not obtained on all patients who died, and that they were not carried out "blind." There may have been deaths due to pulmonary embolism after patients had left hospital. However, despite these reservations, this trial probably represents the best documented evidence for benefit in the whole field of the prevention and treatment of thrombosis. For the group of patients studied-patients over 40 having major surgery-the balance of benefit from low-dose heparin seems clear.

At present the sodium salt is the only form of heparin routinely available in Britain and it is doubtful if a trial to compare the sodium and calcium salts would be justified. As regards patients under 40, heparin should perhaps be restricted to those presumably at risk-the obese, patients with malignant disease, and those with varicose veins or a previous history of deep vein thrombosis, or where lengthy operation or a difficult postoperative course with delay in ambulation are expected. In this trial there was no laboratory control of the heparin therapy, and in view of the relative complexity of the laboratory methods needed (such as Denson and Bonnar ${ }^{20}$ ) the potential benefit of laboratory control-a possible reduction in the incidence of wound haematomas in the heparin group-does not seem to justify the difficulties which would be caused by individual dosage schedules.

How does low-dose heparin compare with other methods of prophylaxis? In 1959 Sevitt and Gallacher ${ }^{21}$ found in a controlled trial that oral anticoagulants were effective in reducing the incidence of both deep vein thrombosis and fatal pulmonary embolism in patients with fractures of the neck of femur; but in a controlled trial of low-dose heparin against nicoumalone in surgical patients ${ }^{22}$ only one of 50 heparin treated patients developed a positive leg scan compared with nine of 50 given dicoumalone-a significant difference $(\mathrm{P}<0.025)$. While dextran 70 has been shown to be more effective than warfarin in reducing the incidence of positive leg scans in patients having major gynaecological surgery, ${ }^{23}$ it is probably less effective than low-dose heparin; in a collaborative study in six hospitals in Edinburgh there was a $37 \%$ incidence of positive leg scans in 128 control patients, $25 \%$ in 130 dextran treated patients, and only $12 \%$ in 128 patients treated with heparin. ${ }^{24}$ In a recent double-blind trial with 831 patients seven control patients died of pulmonary embolism but only one dextran treated patient died ${ }^{25}$; perioperative bleeding in the patients given dextran was not a problem.

Intermittent calf compression during surgery is effective in reducing the incidence of positive leg scans, presumably because of reduced venous stasis during surgery. ${ }^{26} 27$ As reported on page 458 of this issue, Roberts and Cotton have examined the possibility of greater protective effects when low-dose heparin, given for seven days, was added to intermittent calf compression during surgery; disappointingly no additive effect was found. Intermittent calf compression is a simple inexpensive procedure without risks of perioperative bleeding; while it has been shown to be effective in reducing the incidence of positive leg scans the necessary trial has not been carried out to demonstrate an effect on the incidence of pulmonary embolism. Electrical calf muscle stimulation during surgery also reduces the incidence of positive leg scans, but again no data are available on an effect on the incidence of pulmonary embolism. ${ }^{28}$ Rosenberg et al. ${ }^{29}$ found that neither calf muscle stimulation nor low-dose heparin were effective in reducing the incidence of positive leg scans in patients undergoing open bladder and prostatic surgery; in laparotomies for malignant diseases low-dose heparin was effective but calf muscle stimulation was not; in the laparotomies for non-malignant conditions both heparin and calf muscle stimulation were effective.

On the evidence at present available, low-dose heparin, oral anticoagulants, dextran 70, intermittent calf compression, and calf muscle stimulation all probably reduce the incidence of deep vein thrombosis. Only low-dose heparin, oral anticoagulants, and dextran 70 have been adequately shown to reduce the incidence of fatal pulmonary embolism, and as lowdose heparin is probably more effective than dextran ${ }^{24}$ and oral anticoagulants ${ }^{22}$ in the prevention of deep vein thrombosis, and can probably be given without laboratory control, the balance of evidence at present is in favour of low-dose heparin in patients over 40 having major surgery. Widespread use of low-dose heparin is now justified for such patients.

As reflected in the increased incidence of wound haematomas (though not in other measures of perioperative blood loss), a minor degree of impairment of haemostasis in some patients will probably be an inevitable price for the reduced incidence of thromboembolism. To prevent overdosage and minimize bleeding, administration should probably be from single dose ampoules.

A large scale multi-centre trial to compare in surgical patients the effects of sodium heparin and dextran in the prevention of fatal pulmonary embolism is now needed. In addition, an assessment of the value of sodium heparin in the prophylaxis of pulmonary embolism in medical patients, in whom dextran therapy would be inappropriate, is also now called for; both such studies seem appropriate fields of endeavour for the Medical Research Council.

${ }^{1}$ De Takats, G., fournal of the American Medical Association, 1950, 142, 527.

2 Sharnoff, J. G., and DeBlasio, G., Lancet, 1970, 2, 1006.

3 Yin, E. T., Wessler, S., and Stoll, P. J., Fournal of Biological Chemistry, $1970,246,3703$.

4 Rosenberg, R. D., and Damus, P. S., fournal of Biological Chemistry, 1973, 248, 6490 .

5 Rosenberg, R. D., Thrombosis et Diathesis Haemorrhagica, 1974, 33, 51.

6 Yin, E. T., and Wessler, S., Thrombosis et Diathesis Haemorrhagica, 1968, $20,465$.

7 Wessler, S., and Yin, E. T., Circulation, 1973, 47, 671.

${ }^{8}$ Negus, D., et al., British Fournal of Surgery, 1968, 55, 835.

9 Kakkar, V. V., et al., Lancet, 1971, 2, 669.

10 Williams, H. T., Lancet, 1971, 2, 950.

11 Nicolaides, A. N., et al., Lancet, 1972, 2, 890.

12 Ballard, R. M., et al., Fournal of Obstetrics and Gynaecology of the British Commonwealth, 1973, 80, 469.

13 Gallus, A. S., et al., New England fournal of Medicine, 1973, 288, 545.

14 Warlow, C., et al., Lancet, 1973, 2, 934.

15 Steffensen, K. A., Acta Medica Scandinavica, 1969, 186, 519.

${ }^{16}$ Handley, A. J., Lancet, 1972, 2, 623.

17 Browse, N. L., Clemenson, G., and Croft, D. N., British Medical fournal, 1974, 1, 603.

${ }^{18}$ Lahnborg, G., et al., Lancet, 1974, 1, 329

19 International Multicentre Trial, Lancet, 1975, 2, 45.

20 Denson, K. W. E., and Bonnar, J., Thrombosis et Diathesis Haemorrhagica, 1973, 30, 471 .

21 Sevitt, S., and Gallagher, N. G., Lancet, 1959, 2, 981.

22 Vroonhoven, T. J. M. V., van, Zijl, J. van, and Muller, H., Lancet, 1974, $1,375$.

${ }^{23}$ Lambie, J. M., et al., British Medical fournal, 1970, 2, 144

24 Multi-Unit Controlled Trial, Lancet, 1974, 2, 118. 
25 Kline, A., et al., British Medical fournal, 1975, 2, 109.

26 Sabri, S., Roberts, V. C., and Cotton, L. T., British Medical fournal, 1971, 3, 82.

27 Hills, N. H., et al., British Medical fournal, 1972, 1, 131.

28 Browse, N., and Negus, D., British Medical fournal, 1970, 3, 615.

29 Rosenberg, I. L., Evans, Mary, and Pollock, A. V., British Medical fournal, $1975,1,649$

\section{Injuries Round the Patella}

Damage to the extensor mechanism of the knee may occur proximal to the patella, through the patella, or distal to it.

Disruption of the quadriceps muscles mostly occurs in older people after a stumble or fall. It results in complete loss of extension of the knee, and surgery is essential to repair the damaged muscle. Immobilization of the knee in plaster-ofParis postoperatively is required to allow healing, and a long period of rehabilitation is necessary.

Distal to the patella, the injury may occur in two places, the tibial tubercle and the patella ligament. The mildest form of damage to the tibial tubercle, Osgood Schlatter's disease, is either an osteochondritis or a "sprain" of the bone due to repeated severe traction on the growing bone in athletic young people. Partial avulsion of the tibial tubercle is a more severe form of the same injury, in which the tibial tubercle is pulled proximally but not completely avulsed from the tibia. The severest form is complete avulsion of the tibial tubercle. In all three types the patients are young-around puberty. Osgood Schlatter's disease should be treated according to the severity of symptoms; most cases require no more than reassurance and a warning that vigorous exercise will produce pain afterwards. Very painful knees may require a period of rest, possibly even in plaster-of-Paris, to relieve the pain, as might the knees in patients with a partial avulsion of the tubercle. Total avulsion of the tubercle requires surgery to replace and secure it, followed by a period of plaster to allow healing. The epiphysis closes postoperatively and recurvatum of the knee may occur if the patient still has some time left in which to continue growing. Rupture of the patella ligament also occurs in younger patients; it requires surgical repair, with immobilization in plaster to allow healing.

The patella is a sesamoid bone within the quadriceps mechanism and is mainly responsible for increasing the mechanical advantage of those muscles. Fractures of the bone or its removal interfere with that function. Fractures of the patella occur generally in middle-aged patients as a result of a stumble. Patients of all ages may fracture their patellas from direct violence-a problem of practical importance because of its frequency after road accidents.

The management of the fracture differs with the cause. Direct violence usually produces a chip fracture or a stellate fracture. (A chip fracture may be falsely suspected in patients with a bipartate patella; but the absence of a haemarthrosis, the smooth edges of the fragment, and the fact that a similar change is apparent on radiographs of the other knee help to establish the presence of the congenital anomaly.) The haemarthrosis of the knee associated with these fractures is responsible for severe pain and swelling in the joint, and aspiration of the swelling can do much to relieve the patient's symptoms. If the fracture is compound, treatment should be along the usual lines, with wound toilet and antibiotics. Closed fractures of the patella should be treated after aspiration with either a Robert Jones bandage or a plaster-of-Paris cylinder, but with early mobilization to mould the fragments.
If an uneven surface results the patient will get pain and later osteoarthrosis, and late patellectomy will be necessary to relieve the symptoms.

Sudden quadriceps contraction with the knee flexed may cause a transverse fracture of the patella or a fracture of the lower pole. In both instances the quadriceps mechanism is interfered with, especially if there is wide displacement of the fragments. The treatment of the transverse fracture is generally by patellectomy and repair of the quadriceps mechanism. The fracture fragments can be held together by a screw or a wire suture either through the fragments or around them; but attempts to fix the fracture in this way are best reserved for young people. Ideally these surgical procedures will preserve the patella, and quadriceps power will be maintained, but unless the fracture fragments are perfectly reduced pain and later osteoarthrosis will occur, and patellectomy will become essential. Fractures of the lower pole are treated by excision of the fragment and resuture of the patella tendon. Plasterof-Paris immobilization is essential to allow healing to take place.

Sanderson ${ }^{1}$ has recently tried to evaluate the different forms of treatment-though fractures of different types and occurring after different degrees of violence require different management. He reviewed 300 patients treated in the decade 1959-69: $51 \%$ had sustained fractures in road traffic accidents, and the types of fracture were divided equally among transverse, comminuted, and polar.

Those patients with fractures requiring conservative treatment did well; $83 \%$ were in the good to excellent group. Of the patients whose patellas were excised in part or totally $64 \%$ also did well in spite of some loss of power. Those patients whose fractures were fixed internally did less well, only $55 \%$ having satisfactory results. Speed of recovery, measured by time to return to work, varied from a few weeks to some months, but it depended more on the type of job and the patient's motivation than the type of fracture. The most common problems were found to be loss of some range of movement and pain on kneeling. Sanderson's conclusion was that if there is any doubt about treatment, patellectomy is the treatment of choice, in spite of the inevitable loss of power.

1 Sanderson, M. C., Australia and New Zealand fournal of Surgery, 1975, $45,4^{\circ}$

\section{E.M.A.S. Gets Going}

Established on 1 February 1973, the Employment Medical Advisory Service has now got into its stride and issued its first report. ${ }^{1}$ By the end of 1974 the staff comprised 108 coctors and 64 nurses based on about 50 centres in England, Wales, and Scotland. It also receives the help of about $\mathbf{4 5 0}$ Appointed Doctors in carrying out statutory medical examinations. The infant service seems to be flourishing, therefore, but some inner doubts remain.

To what extent occupational health is a specialty in the sense that cardiology, neurology, and surgery are has sometimes been questioned. If it is, what is its scope ?' ${ }^{2}$ Dr. Suzette Gauvain, who has been acting head of the E.M.A.S. since Dr. T. A. Lloyd Davies retired, is able to report that relations with other workers and organizations in occupational medicine have been cordial. But the importance of this branch of medicine "is unfortunately not always recognized." The 